PHYSICAL REVIEW B 90, 094402 (2014)

\title{
Temperature-dependent ferromagnetic resonance via the Landau-Lifshitz-Bloch equation: Application to FePt
}

\author{
T. A. Ostler and M. O. A. Ellis \\ Department of Physics, University of York, Heslington, York YO10 5DD, United Kingdom \\ D. Hinzke and U. Nowak \\ Fachbereich Physik, Universität Konstanz, D-78457 Konstanz, Germany \\ (Received 4 July 2014; revised manuscript received 14 August 2014; published 2 September 2014)
}

\begin{abstract}
Using the Landau-Lifshitz-Bloch (LLB) equation for ferromagnetic materials, we derive analytic expressions for temperature-dependent absorption spectra as probed by ferromagnetic resonance. By analyzing the resulting expressions, we can predict the variation of the resonance frequency and damping with temperature and coupling to the thermal bath. We base our calculations on the technologically relevant $\mathrm{L} 1_{0} \mathrm{FePt}$, parametrized from atomistic spin dynamics simulations, with the Hamiltonian mapped from $a b$ initio parameters. By constructing a multimacrospin model based on the LLB equation and exploiting GPU acceleration, we extend the study to investigate the effects on the damping and resonance frequency in $\mu \mathrm{m}$-sized structures.
\end{abstract}

DOI: 10.1103/PhysRevB.90.094402 PACS number(s): 75.70.-i, 75.50.Bb, 75.50.Vv, 75.78. $-\mathrm{n}$

\section{INTRODUCTION}

The magnetic properties of ferromagnetic structures such as thin films, nanowires, and nanoparticles have been studied extensively both experimentally [1,2] and theoretically [3,4]. The interest in these particles is driven by fundamental features on the one hand and technological perspectives on the other [5-7]. Ferromagnetic resonance (FMR), which has been applied with great success to thin ferromagnetic films in the past [8], can be used to measure important material properties, such as the damping, gyromagnetic ratio, and anisotropy constant. The temperature dependence of these properties for large or complex structures is often difficult to predict using analytical treatments, especially when temperature effects are included [4,9]. As well as being difficult to calculate analytically, temperature-dependent calculations of (for example) FMR can be slow to converge. The convergence can become particularly troublesome if thermal fluctuations are accounted for. A specific motivation for this work is the interest in $\mathrm{L} 1_{0} \mathrm{FePt}$ materials, which is a promising candidate for ultrahigh density magnetic recording $[10,11]$.

The ability to tune magnetic properties such as the damping is important, for example, in devices based on spin-transfer torque where a low damping of a free layer is essential for reducing the power consumption and can affect the signal-tonoise ratio [12]. In some cases, such as in giant magnetoresistive (GMR) read sensors, high damping is preferred to improve thermal stability [13].

For technologies based on heat-assisted magnetic recording (HAMR), understanding temperature effects and fluctuations in strongly anisotropic materials will be crucially important. In this paper, we present analytical and numerical calculations of the material properties of strongly anisotropic materials at elevated temperatures. We do so by utilizing the formalism of the Landau-Lifshitz-Bloch (LLB) equation of motion for ferromagnetic particles, which has an intrinsic temperature dependence via various input functions. There are a number of different approaches to calculating FMR and introducing temperature effects. The work of Usadel [14] utilizes an approach based on the Landau-Lifshitz-Gilbert (LLG) equation for nanoparticles whereby ensembles of atomic spins are treated as a single macrospin, in the same manner as the Landau-Lifshitz-Bloch equation. However, the work presented in Ref. [14] does not take into account the contraction of the magnetization length. As the LLG model does not take into account the longitudinal relaxation of the magnetization, which becomes important at elevated temperatures, there is a requirement to use an approach such as the LLB to correctly describe the temperature-dependent properties as we approach the Curie temperature.

Other approaches for numerical determination of FMR properties in systems where exchange between macrospins is important, such as thin films or granular media, including micromagnetic simulations such as that of Ref. [3]. In Ref. [3] the study focuses on granular media with the exchange between macrospins within a grain and between grains taken into account. The use of this kind of micromagnetic model is well accepted at temperatures clearly below the Curie temperature where the magnitude of the magnetization is determined by the temperature. However, at higher temperatures the susceptibility increases and due to thermal fluctuations the magnetization locally cannot be regarded as constant [15]. The use of the LLB model is of greatest importance when the susceptibility begins to increase and small variations in temperature result in large changes in magnetization (around $T \gtrsim 3 T_{\mathrm{C}} / 4$ ) [16].

In the first part of the paper, we present the derivation of the temperature-dependent analytic expression for the power absorbed by the particle. This analytic expression allows us to look at the effect of temperature on FMR curves for single-domain particles. The temperature-dependent input functions that enter into the LLB formalism have been parametrized from atomistic spin dynamics with the exchange parameters calculated directly from $a b$ initio calculations [17]. We have tested the expressions with a single-spin and multispin (with exchange) LLB numerical model, by showing a number of resonance curves at different temperatures against the derived expressions (without demagnetizing fields). The analytic expressions for the damping and resonance 
frequency show the overall trend of the temperature-dependent behavior.

In the second half of the paper, we extend the scope of our analysis using a multimacrospin model based on the LLB formalism with large number of exchange-coupled macrospins. We present numerical calculations of FMR in two-dimensional (2D) and three-dimensional (3D) structures with the inclusion of demagnetizing effects and (stochastic) thermal fluctuations. Specifically, we have investigated the effects of the anisotropy constant and film thickness and anisotropy on the measured damping in out-of-plane films at high temperatures. Our findings show that, depending on thickness or anisotropy, there is a competition between the demagnetizing and anisotropy energy that can modify the damping significantly. We have implemented this large-scale model on the CUDA GPU platform so that even with the inclusion of the stochastic thermal terms, it is possible to obtain good averaging of the FMR power spectra.

There are limited experimental ferromagnetic studies of chemically ordered FePt due to its large magnetocrystalline anisotropy [18]. However, it is possible to perform so-called optical FMR with the use of laser pulses [19]. In a theoretical work by Butera [20], the resonance spectra were calculated using a computational model for disordered nanoparticles of FePt. This study showed that the measured damping depended strongly on the amount of disorder. To our knowledge, there are no systematic studies on the temperature dependence of the properties such as damping due to the limited fields in typical FMR setups; our results provide insight into this complex issue.

\section{LANDAU-LIFSHITZ-BLOCH EQUATION}

The LLB equation for magnetic macrospins describes the time evolution of an ensemble of atomic spins and allows for relaxation of the magnitude of the magnetization. The equation was originally derived by Garanin [21] within a mean-field approximation from the classical Fokker-Planck equation for atomistic spins interacting with a heat bath. The resulting LLB equation has been shown to be able to describe linear domain walls, a domain-wall type with nonconstant magnetization length. These results are consistent with measurements of the domain-wall mobility in yttrium iron garnet (YIG) crystals close to the Curie point $\left(T_{\mathrm{c}}\right)[22]$ and with atomistic simulations [23]. Furthermore, the predictions for the longitudinal and transverse relaxation times have been successfully compared with atomistic simulations [24]. Consequently, we use this equation in the following for the thermodynamic simulations of macrospins. The use of the LLB formalism has the advantage over traditional micromagnetics that it automatically allows for changes in the modulus of the magnetization. In theory, it is indeed possible to calculate temperature-dependent FMR using the atomistic spin dynamics (ASD) model, however, such an approach would be extremely computationally expensive. This computational expense in the ASD model arises because, for FMR calculations, large system sizes are required to reduce the effects of thermal noise. While large systems are possible to calculate, the FMR calculations also require averaging over many cycles of the driving field, up to hundreds of nanoseconds. These two restrictions combined means that this method is not suitable, even with GPU acceleration or a (for example, MPI) distributed memory solution [25].

A further challenge for accurate calculation of magnetic properties is the accounting of the long-ranged exchange in materials such as FePt. Through proper parametrization of the LLB equation [17], one can account for such long-ranged interactions in the so-called multiscale approach [17]. Via this multiscale approach we can then bridge the gap between electronic-structure calculations to large-scale (of the order of micrometres) calculations of material properties. With this in mind, the LLB model is then ideally placed to describe temperature-dependent ferromagnetic resonance.

The LLB equation, without the stochastic term, can be written in the form

$$
\begin{aligned}
\dot{\mathbf{m}}= & -\gamma\left[\mathbf{m} \times \mathbf{H}_{\mathrm{eff}}\right]+\frac{\gamma \alpha_{\|}}{m^{2}}\left(\mathbf{m} \cdot \mathbf{H}_{\mathrm{eff}}\right) \mathbf{m} \\
& -\frac{\gamma \alpha_{\perp}}{m^{2}}\left[\mathbf{m} \times\left[\mathbf{m} \times \mathbf{H}_{\mathrm{eff}}\right]\right] .
\end{aligned}
$$

Aside from the usual precession and relaxation terms, the LLB equation contains another term which controls longitudinal relaxation [second term in Eq. (1)]. Hence, $\mathbf{m}$ is a spin polarization which is not assumed to be of constant length and even its equilibrium value $m_{\mathrm{e}}(T)$ is temperature dependent. The value of $\mathbf{m}$ is equal to the ratio of the magnetization of the macrospin normalized by the magnetization at saturation $\left(\mathbf{M} / M_{\mathrm{S}} V\right) . \alpha_{\|}$and $\alpha_{\perp}$ are dimensionless longitudinal and transverse damping parameters (defined below) and $\gamma$ is the gyromagnetic ratio taken to be the free-electron value. The transverse damping parameter in this equation is related to what is usually measured in experiments (the Gilbert damping $\alpha_{\mathrm{g}}$ ) by the expression

$$
\alpha_{\mathrm{g}}=\frac{\alpha_{\perp}}{m} .
$$

The LLB equation is valid for finite temperatures and even above $T_{\mathrm{c}}$, although the damping parameters and effective fields are different in the two regions. Throughout this paper, we are only interested in the case $T \leqslant T_{\mathrm{c}}$ with the damping parameters [21] $\alpha_{\|}=\frac{2 \lambda T}{3 T_{\mathrm{c}}}$ and $\alpha_{\perp}=\lambda\left(1-\frac{T}{3 T_{\mathrm{c}}}\right)$. The singleparticle free energy (without demagnetizing fields) is given by

$$
f=-B M_{\mathrm{s}}^{0} m_{z}+\frac{M_{\mathrm{s}}^{0}}{2 \tilde{\chi}_{\perp}}\left(m_{x}^{2}+m_{y}^{2}\right)+\frac{M_{\mathrm{s}}^{0}}{8 \tilde{\chi}_{\|} m_{e}^{2}}\left(m^{2}-m_{e}^{2}\right)^{2},
$$

and the effective fields $\mathbf{H}_{\text {eff }}=-\frac{1}{M_{\mathrm{s}}} \frac{\delta f}{\delta \mathbf{m}}$ given by [21]

$$
\mathbf{H}_{\mathrm{eff}}=\mathbf{B}+\mathbf{H}_{A}+\frac{1}{2 \tilde{\chi}_{\|}}\left(1-\frac{m^{2}}{m_{\mathrm{e}}^{2}}\right) \mathbf{m},
$$

where $\mathbf{B}$ represents an external magnetic field and the anisotropy field $\mathbf{H}_{A}$ is given by

$$
\mathbf{H}_{A}=-\left(m_{x} \mathbf{e}_{x}+m_{y} \mathbf{e}_{y}\right) / \tilde{\chi}_{\perp} .
$$

Here, the susceptibilities $\tilde{\chi}_{l}$ are defined by $\tilde{\chi}_{l}=\partial m_{l} / \partial H_{l}$, where $H_{l}$ is the $l=\|, \perp$. In these equations, $\lambda$ is a microscopic parameter which characterizes the coupling of the individual, 
atomistic spins with the heat bath. The anisotropy field $\mathbf{H}_{A}$ [Eq. (5)] defines a hard axis in the $x$ and $y$ planes, essentially giving a uniaxial anisotropy in the $z$ direction. This allows the anisotropy field to be defined in terms of the transverse susceptibility [17] $\left(\chi_{\perp}\right)$ and gives the correct scaling of the anisotropy [26].

For the purpose of testing the model, we use a thermal bath coupling constant of $\lambda=0.05$, consistent with Ref. [18]. There are differing values of the damping constant in the literature, for example, for granular FePt Becker et al. measured a damping constant of 0.1 using an optical FMR technique, whereas Alvarez et al. found a value of 0.055 using standard FMR in a broad frequency range [18]. It should be pointed out here that while $\lambda$ is a coupling to the thermal bath equivalent to that used in atomistic spin dynamics, it is assumed to be temperature independent.

At this point, we should take some time to define the different constants related to the damping and their differences. The parameters $\lambda, \alpha_{\perp}, \alpha_{\|}$, and $\alpha_{\mathrm{g}}$ correspond to the thermal bath coupling, the temperature-dependent transverse and longitudinal damping parameters, and the damping parameter that one would measure experimentally, respectively. The thermal bath coupling is temperature independent and is a phenomenological parameter that is the same as that used in atomistic spin dynamics. The transverse and longitudinal damping parameters that enter into the LLB equation define the relaxation rates of the transverse and longitudinal magnetization components. Finally, the parameter $\alpha_{\mathrm{g}}$ is equal to the perpendicular damping $\left(\alpha_{\perp}\right)$ that enters into the equation of motion, divided by the magnetization and is what one would find in an FMR measurement from the linewidth.

For the application of this equation, one has to know $a$ priori the spontaneous equilibrium magnetization $m_{\mathrm{e}}(T)$ and the perpendicular $\left[\tilde{\chi}_{\perp}(T)\right]$ and parallel $\left[\tilde{\chi}_{\|}(T)\right]$ susceptibilities. In this work, these are calculated separately from a Langevin dynamics simulation of an atomistic spin model, however, it is possible to calculate these properties from mean-field calculations [27]. We use a model for FePt which was introduced earlier and which is meanwhile well established in the literature [26,28-30]. Since this model was derived from first principles, a direct link is made from spin-dependent density functional theory calculations, via a spin model, to our macrospin simulations. The calculation of these parameters is discussed in more detail in Ref. [17].

\section{ANALYTIC SOLUTION FOR THE FMR ABSORBED POWER SPECTRUM P $(\omega)$}

The focus of this section is on the derivation of an analytical solution for the power spectrum $P(\omega)$ using the LLB equation for a single macrospin. The power $P(\omega)$ absorbed in an FMR experiment is given by [4]

$$
P(\omega)=\left\langle\mathbf{M} \cdot \frac{\partial \mathbf{B}}{\partial t}\right\rangle=-\frac{\omega}{2 \pi} \int_{0}^{2 \pi / \omega} M_{\mathrm{S}} V m_{x} \dot{B}_{x} d t,
$$

where $V$ is the volume of the macrospin, $\mathbf{M}$ is the magnetization $\left(M_{\mathrm{S}} V \mathbf{m}\right)$, and $\omega$ is the frequency of the driving field. The right-hand side of Eq. (6) assumes that the time-varying field is applied in the $x$ direction with the static applied field in $z$. The time dependence of the $x$ component of the magnetization can be derived from Eq. (1). Using the assumptions that $m^{2}$ is constant and $m_{x}$ as well as $m_{y}$ are small leads to the approximation $m_{z} \approx m$. Under this assumption, Eq. (1) can be written in linearized form. Together with the linearized form of the effective field, the solution of the resonance frequency $\omega_{0}$ and transverse relaxation time $\tau$ can be obtained (for full details see Appendix A):

$$
\begin{aligned}
\omega_{0}(T) & =\gamma\left(B_{z}+\frac{m(T)}{\tilde{\chi}_{\perp}(T)}\right), \\
\tau(T) & =\frac{m(T)}{\lambda\left[\left(1-\frac{T}{3 T_{\mathrm{c}}}\right) \omega_{0}(T)-\frac{2}{3} \gamma \frac{T}{T_{\mathrm{c}}} H_{\mathrm{eff}}^{z}(T)\right]} .
\end{aligned}
$$

Here, $m=m_{e}+\tilde{\chi}_{\|} B_{z}$ is an approximation written to first order of the susceptibility for the purposes of the analytic calculation. In the zero-temperature case under the conditions that $\alpha=\alpha_{\perp}, \alpha_{\|}=0$, and $m=m_{\mathrm{e}}=1, \omega_{0}$ and $\tau$ are the same as for the Landau-Lifshitz-Gilbert (LLG) equation $\omega_{0}=$ $\gamma\left(B_{z}+\frac{1}{\tilde{\chi}_{\perp}}\right)$ and $\tau=\frac{1}{\lambda \omega_{0}}$.

The analysis of Eqs. (7) and (8) shows that there is little variation of the measured damping $\alpha_{\mathrm{g}}$ with the applied field as one would expect [31]. Also, at low temperature, as expected, the measured damping is equal to the input coupling to the thermal bath $\lambda$. The temperature dependence of $\alpha_{\mathrm{g}}$ shows that (for a chosen value of $\lambda$ ) there is an increase with temperature, diverging at the Curie point. In a recent paper [31], the measured damping as a function of applied field (up to $7 \mathrm{~T}$ ) was shown to be almost independent of temperature. In the same study, the damping was measured at two temperatures: 170 and $290 \mathrm{~K}$. Between these two temperatures the damping was shown to be around 0.1 with a slight increase as one would expect.

Figure 1 shows the analysis of Eqs. (7) and (8) for the physical input parameters for FePt. In the figure the measured damping is calculated as $\alpha_{g}=1 / \omega_{0} \tau$ and is shown to increase with temperature, diverging at the Curie point. The contours show lines of constant damping explicitly. This demonstrates that if one assumes no temperature dependence of the thermal bath coupling $\lambda$, the measured damping will not be constant.

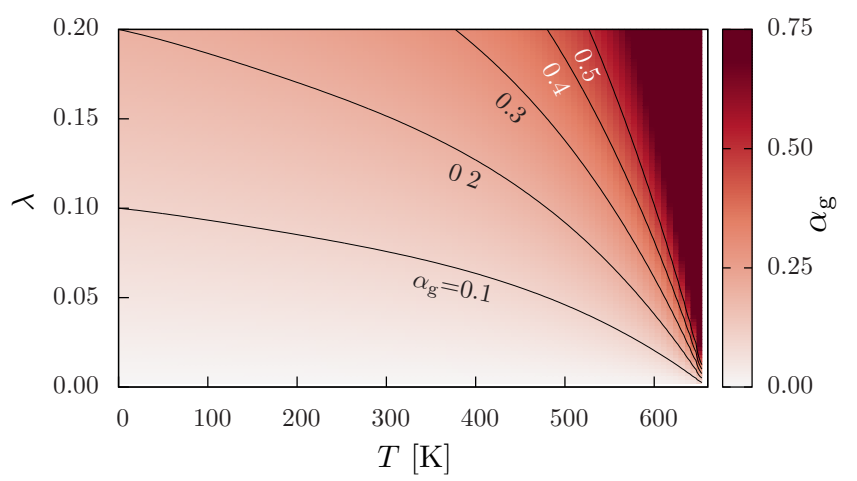

FIG. 1. (Color online) Analytically derived Gilbert damping as a function of temperature and the intrinsic coupling to the thermal bath $\lambda$, valid for a single macrospin without demagnetizing effects. For each value of $\lambda$ the damping is shown to increase with temperature consistent with other works [24,31]. The lines are contours of constant measured damping. 
The value of $\lambda$ shown on the $y$ axis of Fig. 1 is a temperature-independent parameter. As mentioned above, this parameter is equivalent to the coupling/damping parameter used in atomistic spin dynamics models. The general approach for atomistic spin dynamics models is to use a constant value of $\lambda$ which governs the rate of energy transfer to the bath [32]. The overall damping measured in the atomistic model is determined by this rate of energy transfer but is also affected by the presence of spin-wave interactions in the system. The measured damping in atomistic spin dynamics is larger than the coupling to the thermal bath (at elevated temperatures) due to spin-wave broadening.

The LLB equation for a single spin contains the parallel and perpendicular damping constants $\left(\alpha_{\perp}\right.$ and $\left.\alpha_{\|}\right)$. These values depend on $\lambda$ (the microscopic coupling to the bath) and intrinsically give a temperature-dependent damping that was derived via the Fokker-Planck equation for the interacting atomistic spins [21].

The solution of the resulting inhomogeneous differential equation (A4) combined with Eq. (6) leads us to the analytic solution for the power absorbed during ferromagnetic resonance as a function of the frequency of the driving field:

$$
P(\omega, T)=\frac{M_{\mathrm{s}} V \omega^{2}}{4} \frac{\gamma \alpha_{\perp} B_{0}^{2}}{\frac{1}{\tau^{2}}+\left(\omega-\omega_{0}\right)^{2}},
$$

where the temperature dependence comes from $\omega_{0}$ and $\tau$ [see Eqs. (7) and (8)] and $B_{0}$ is the amplitude of the driving field. In the zero-temperature case, this solution reduces to that from the LLG equation. In Fig. 2 (shown and discussed below) the temperature dependence of the analytic solution enters via the temperature-dependent input functions of the LLB equation. The FMR equation given by Eq. (9) (and shown analytically in Fig. 2) uses the functions for FePt that were presented in Ref. [17], however, similar functions could be calculated using mean-field theory [27].

The analytic solution given by Eq. (9) can be compared to the numerical results, by integration of the LLB equation and using Eq. (6). By applying an alternating driving field in the $x$ direction and numerically averaging Eq. (6) until convergence we can compare the results of a single spin to the analytic expression. For FePt, there is a strong uniaxial exchange anisotropy, therefore, in the absence of any static applied field

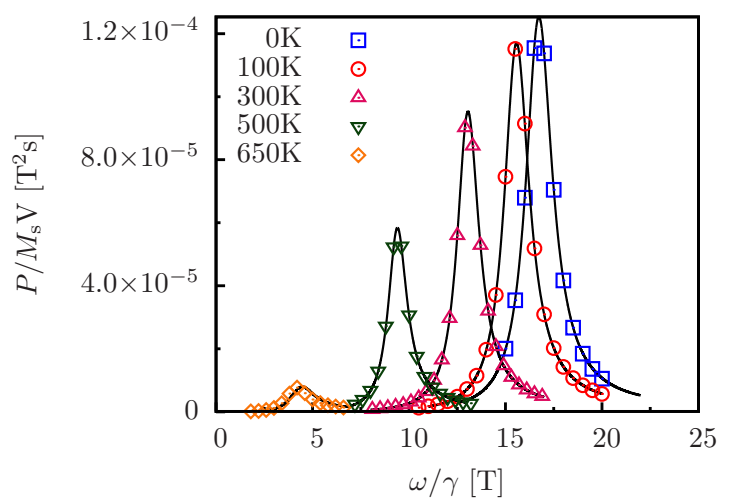

FIG. 2. (Color online) Power spectrum vs frequency in a 1-T applied field. The data points are from LLB simulations for a single macrospin and the solid lines are given by Eq. (9). we still see a very strong FMR line for single-domain particles. Throughout the calculations we use a driving field amplitude $\left(B_{0}\right)$ of $0.005 \mathrm{~T}$ and a static applied field $\left(B_{z}\right)$ of $1 \mathrm{~T}$. The Curie temperature for our system was assumed to be $660 \mathrm{~K}$ [17], the saturation magnetization used was $\mathrm{M}_{\mathrm{s}}=1047785 \mathrm{JT}^{-1} \mathrm{~m}^{-3}$. The anisotropy field at $0 \mathrm{~K}$ is equal to $1 / \tilde{\chi}_{\perp}(0 \mathrm{~K})$ and is equal to $15.69 \mathrm{~T}$, i.e., a value of the anisotropy constant $K\left(\right.$ via $\left.H_{A}=2 K / \mathrm{M}_{\mathrm{s}}\right)$ of $8.2 \times 10^{6} \mathrm{Jm}^{-3}$. The temperature dependence of the transverse susceptibility via Eq. (5) scales the anisotropy with $\mathrm{M}^{2.1}$ as shown in Ref. [26]. In the second part of the paper, we have scaled the anisotropy constant at $0 \mathrm{~K}$ by a given amount to give a different value of the anisotropy constant used.

The reason for using a static applied field and varying the frequency of the driving field is for computational efficiency. In the second part of this work, we have simulated a large number of macrospins coupled via exchange and magnetostatics, which is computationally very expensive. To obtain good averages of the absorbed power during FMR we require a large number of cycles of the driving field. The use of driving frequencies around $10-60 \mathrm{GHz}$ would drastically increase the simulation time, particularly for the low-temperature (highresonance-field) simulations. Therefore, the simulation time for higher frequencies is lower, increasing as it is reduced. The use of a higher-frequency driving field would overcome the computational problem, however, a large static applied field (particularly at low temperature) would be required to drive the system to resonance. Both the high frequency and the high field would be very difficult to obtain experimentally. The expression for the FMR power [Eq. (9)] can also be presented in the form $P\left(B_{z}\right)$. We have shown the analytic curves for this representation in Appendix B, although the quantities derived, such as damping, from the curves in either representation should be consistent.

We integrate the LLB equation using the Heun numerical scheme with a 5-fs time step. The input functions $\left[m_{e}(T)\right.$, $\chi_{\perp, \|}(T)$, and $\left.A(T)\right]$ that were used for FePt [as used in Eq. (9)] were calculated from atomistic spin dynamics [17] and the functional forms are polynomial fits [33]. The exact functions can be found in Ref. [33], specifically on pages 143 and 144 and are the same as those in Figs. 1, 2, and 3 of Ref. [17].

Figure 2 shows the calculated absorbed power as a function of frequency for a range of temperatures using the single-spin LLB model. As we can see from Fig. 2, there is a large decrease in the resonance frequency, given by Eq. (7), which we would expect to occur because of the decrease in the anisotropy field. The analytic solution agrees perfectly with the numeric model, except as we approach the Curie temperature. This is because in the analytical treatment we approximate the magnetization in a field $B_{z}$ to depend on the parallel susceptibility $\left(m=m_{e}+\right.$ $\tilde{\chi}_{\|} B_{z}$ ) which diverges as we approach the Curie temperature. This point has been discussed in Appendix A and is an error in the analytic treatment only, not in the form of the LLB equation.

The reduction in the anisotropy with temperature shown in Fig. 2 is represented by a reduction in the resonance frequency in the $P(\omega)$ representation. As we have shown in Appendix $\mathrm{B}$, in the $P\left(B_{z}\right)$ representation, the resonance field increases with temperature. Both of these representations are consistent with the expected decrease in temperature and are in 
qualitative agreement with other works, for example, the experimental works of Schulz and Baberschke [34] for the case of perpendicular films with the field applied perpendicular to the film. The work of Antoniak [35] on FePt nanoparticles, as well as the theoretical work of Usadel [14] using an LLG-based model for (dipole) interacting nanoparticles, show a similar increase in the resonance field with temperature (reduction in the resonance frequency).

\section{MULTIMACROSPIN NUMERICAL RESULTS}

In the following section, we introduce the stochastic LLB equation that takes into account thermal fluctuations. As well as the normal terms in the LLB described by Eq. (4), we also include exchange coupling between the macrospins and the magnetostatic fields. The LLB equation with stochastic thermal terms included is written for each spin $i$ in the form

$$
\begin{aligned}
\dot{\mathbf{m}}_{i}= & -\gamma\left[\mathbf{m}_{i} \times \mathbf{H}_{\mathrm{eff}}^{i}\right]+\zeta_{i, \|}-\frac{\gamma \alpha_{\perp}}{m_{i}^{2}}\left[\mathbf{m}_{i} \times\left[\mathbf{m}_{i}\right.\right. \\
& \left.\left.\times\left(\mathbf{H}_{\mathrm{eff}}^{i}+\zeta_{i, \perp}\right)\right]\right]+\frac{\gamma \alpha_{\|}}{m_{i}^{2}}\left(\mathbf{m}_{i} \cdot \mathbf{H}_{\mathrm{eff}}^{i}\right) \mathbf{m}_{i} .
\end{aligned}
$$

The stochastic fields $\zeta_{i, \perp}$ and $\zeta_{i, \|}$ have zero mean and the variance [16]

$$
\begin{aligned}
\left\langle\zeta_{i, \perp}^{\eta}(0) \zeta_{j, \perp}^{\theta}(t)\right\rangle & =\frac{2 k_{\mathrm{B}} T\left(\alpha_{\perp}-\alpha_{\|}\right)}{|\gamma| M_{s} V \alpha_{\perp}^{2}} \delta_{i j} \delta_{\eta \theta} \delta(t), \\
\left\langle\zeta_{i, \|}^{\eta}(0) \zeta_{j, \|}^{\theta}(t)\right\rangle & =\frac{2|\gamma| k_{\mathrm{B}} T \alpha_{\|}}{M_{s} V} \delta_{i j} \delta_{\eta \theta} \delta(t),
\end{aligned}
$$

where $\|$ is the additive noise, and $\eta$ and $\theta$ represent the Cartesian components. As well as the stochastic field, the exchange is also included in the form

$$
\mathbf{H}_{e x}^{i}=\frac{A(T)}{m_{e}^{2}} \frac{2}{M_{s}^{0} \Delta^{2}} \sum_{j \in \operatorname{neigh}(i)}\left(\mathbf{m}_{j}-\mathbf{m}_{i}\right),
$$

where $A(T)$ is the exchange stiffness, $\Delta$ is the cell length, and $M_{s}^{0}$ is the saturation magnetization. It should be pointed out that the inclusion of the stochastic term into the LLB equation leads to a slightly reduced $T_{\mathrm{C}}$ as compared to the LLB without the stochastic term [16].

Figure 3 shows the power spectrum as a function of frequency for multimacrospin calculations (coupled by exchange) for a system size of $(100 \mathrm{~nm})^{3}$ with a unit-cell discretization of $(6.25 \mathrm{~nm})^{3}$ (i.e., $16 \times 16 \times 16$ macrospins), though we have checked unit-cell sizes down to $(3.125 \mathrm{~nm})^{3}(32 \times 32 \times 32$ macrospins), i.e., below the typical domain-wall size of 4 $6 \mathrm{~nm}$. The solid lines are the analytical solution (9). The macrospin lattice is represented as a simple cubic arrangement with only nearest-neighbor interaction taken into account and is the same for all simulations involving many macrospins.

As discussed in the Introduction, we have also introduced into our model demagnetizing effects to extend the analytic study to more realistic materials. We have taken the approach of that of Lopez-Diaz et al. used in the GPMAGNET software [36]. In this approach, we write the magnetostatic field in a (cubic) cell $i\left(\mathbf{H}_{d, i}\right)$ as

$$
\mathbf{H}_{d, i}=-M_{s} \sum_{j} \mathbf{N}\left(\mathbf{r}_{i}-\mathbf{r}_{j}\right) \cdot \mathbf{m}_{j},
$$

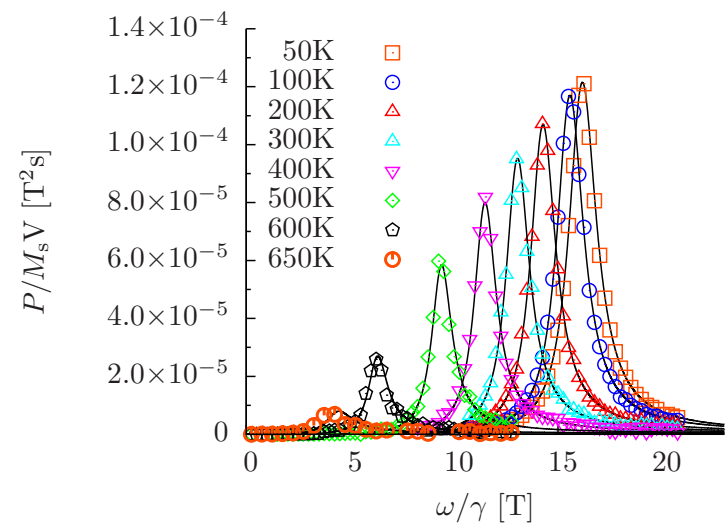

FIG. 3. (Color online) Power spectrum vs frequency in a 1-T applied field. The data points are from LLB simulations for many exchange-coupled macrospins including the stochastic fields and exchange and the solid lines are given by Eq. (9) (no magnetostatic fields are included here).

where $\mathbf{N}$ is the $3 \times 3$ symmetric demagnetizing tensor. The sum runs over all cells at positions $\mathbf{r}_{i, j}$. The demagnetizing tensor is given by

$$
\mathbf{N}\left(\mathbf{r}_{i}-\mathbf{r}_{j}\right)=\frac{1}{4 \pi} \oint_{S_{i}} \oint_{S_{j}} \frac{d \mathbf{S}_{i} \cdot d \mathbf{S}_{j}^{\prime}}{\left|\mathbf{r}-\mathbf{r}^{\prime}\right|},
$$

$S_{i}\left(S_{j}\right)$ are the surface of cell $i(j)$, respectively, $\mathbf{r}$ and $\mathbf{r}^{\prime}$ are the points on the surface $i$ and $j$. This sum requires a summation from all cells and requires integration over each of the surfaces $i$ and $j$, making it extremely computationally expensive. If one were to perform the integration (14) numerically for each surface of each cell, the calculation is extremely time consuming and converges very slowly with the number of mesh points on each surface. To that end, we have employed the method of Newell [37], whereby the surface integration of the cubes is calculated analytically as in the OOMMF code [38]. Some further details can be found in Appendix C.

\section{FERROMAGNETIC RESONANCE IN THIN FILMS OF FEPT}

In this section, we present calculations of thin films of FePt. We begin by looking at the effect of temperature on the damping of 2-nm thin films using the stochastic form of the LLB equation with demagnetizing fields [Eq. (13)]. We compare this to the results for the single-spin analytic results. The thin films show a large increase in the predicted damping over the analytic results due to the inclusion of the demagnetizing term as we approach the Curie temperature. The thickness dependence of the films is also calculated using the multispin model, showing that at low temperatures there is little variation in damping with film thickness though, at temperatures approaching the Curie point, there is a large reduction with increasing thickness.

For the thin films of FePt where we have included the magnetostatic interactions into the system, the analytic form of the resonance curves can no longer be fitted to the data. We therefore extract the damping by fitting the following 


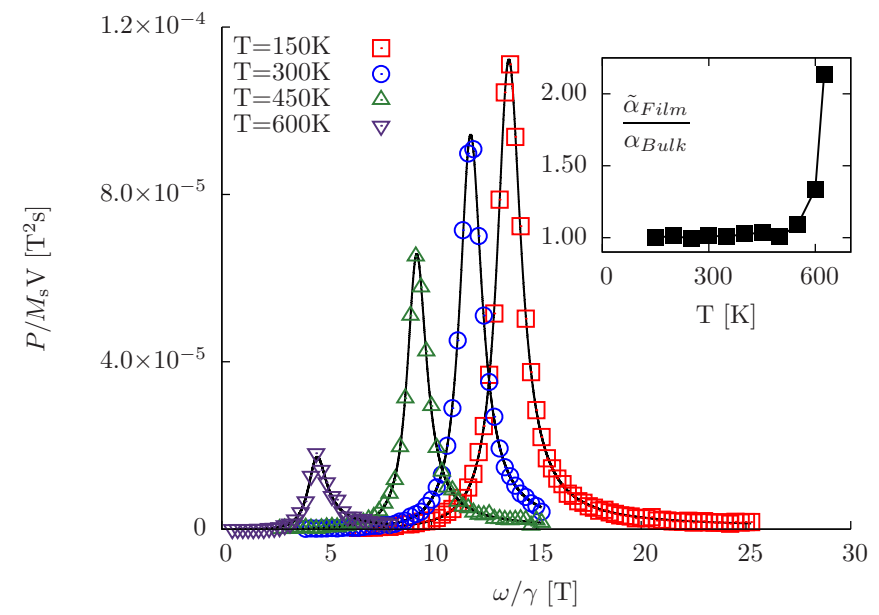

FIG. 4. (Color online) Ferromagnetic resonance curves in thin films of FePt for a range of temperatures below the Curie temperature. The points here are simulated data and the lines are the fits to Eq. (15). The inset shows the ratio of the damping as measured in our 2D film to the damping calculated analytically for a single macrospin. For low temperatures, the two are equivalent, however, at higher temperatures there is an enhanced damping in the thin films due to the effect of the magnetostatic field.

expression to the FMR curves:

$$
P(\omega)=P_{0} \frac{\omega^{2}}{\left(\omega \tilde{\alpha}_{g}\right)^{2}+\left(\omega-\tilde{\omega}_{0}\right)^{2}},
$$

where $\tilde{\alpha}_{g}, P_{0}$, and $\tilde{\omega}_{0}$ are free-fitting parameters and we use the tilde to distinguish the resonance frequency and damping from the analytically derived values. The use of this fitting procedure allows us to compare with experimental observations as this would be the kind of analysis required to extract the damping parameter $\left(\alpha_{\mathrm{g}}\right)$. For the single-spin calculations (as shown in Sec. III), we have verified that the use of this expression recovers the analytic value of the damping $\alpha_{g}$.

By systematically varying the anisotropy we have shown that this increase in damping occurs when the demagnetizing field dominates over the anisotropy term. Finally, this modification in the damping is shown to affect the switching times as we transition from one regime to another.

The $x$ and $y$ dimensions of the thin films in this section are $0.4 \mu \mathrm{m} \times 0.4 \mu \mathrm{m}$. The $z$ dimension is initially one cell $(2 \mathrm{~nm})$ thick, i.e., a 2D film. Our cell discretization is $2 \mathrm{~nm} \times 2 \mathrm{~nm} \times$ $2 \mathrm{~nm}$, below the domain-wall width. We apply the fields in the same orientation as discussed above. The resonance curves are shown on Fig. 4 for a range of temperatures for the 2D (2-nm-thick) film. From each FMR curve we have used a fitting procedure, as in Fig. 2, to calculate the damping in the 2D structures (solid lines). The inset of Fig. 4 shows then the ratio of the damping that we calculate for the 2D structures to the analytically derived damping for single-domain particles in Sec. III. In the low-temperature limit, this ratio is consistent with the analytic solution for a single macrospin (i.e., it is 1); for high temperature, however, the damping is increased as the demagnetizing effects start to dominate over the anisotropy.

Next, we consider the effects of film thickness on the damping and resonance frequency. We increase the thickness

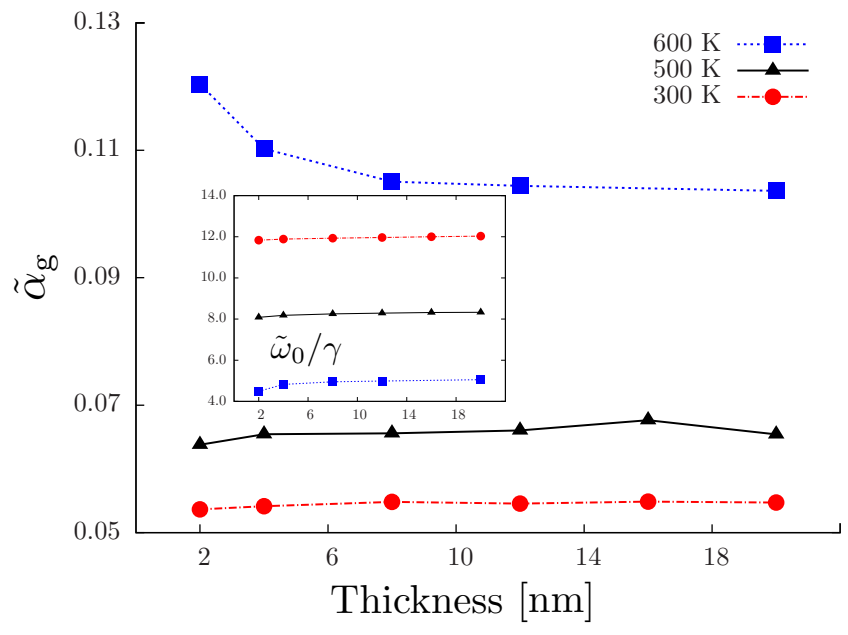

FIG. 5. (Color online) Damping as a function of film thickness for a range of temperature. In the low-temperature regime, there is a slight increasing damping as a function of thickness. As the Curie temperature is approached, there is a large decrease in the damping with film thickness. The inset shows the variation of the resonance frequency with thickness. The resonance frequency shows an overall increase over all temperatures due to the decrease in the effective magnetostatic field.

of the film from 2 to $20 \mathrm{~nm}$ ( 1 to 10 cells) and calculate the ferromagnetic resonance curve for each thickness (a maximum of 400000 cells for around $100 \mathrm{~ns}$ ). The resulting FMR curves were again analyzed to extract the damping and resonance frequencies.

Figure 5 shows the variation of the damping and resonance frequency as a function of the thickness of the thin film. The largest variation in the damping is shown close to the Curie temperature (blue square, dotted line). For $T=$ $500 \mathrm{~K}$, there is a small increase in the damping with film thickness when going from 2 to $4 \mathrm{~nm}$. After $4 \mathrm{~nm}$, the curve shows little variation, consistent with the $T=300 \mathrm{~K}$ (red circles, dotted-dashed line) line. The variation in the damping, with film thickness, close to the Curie point will have a large effect on the magnetization dynamics in heat-assisted magnetic recording (for which $\mathrm{FePt}$ is a promising candidate), that operates at elevated temperatures. The elevated temperatures allow for the reduction in the anisotropy so that the field generated by the write head of a hard disk drive (around 1-2 T) is sufficient to reverse the magnetisation. This reduction in damping for thick layers of $\mathrm{FePt}$ would lead to longer switching times (as we show in the following), limiting the write times.

In Ref. [39], Liu et al. showed that the damping in a magnetic tunnel junction consisting of a $\mathrm{FeCoB}$ free layer increased with decreasing thickness. The mechanism was said to be caused by spin pumping and nonlocal background effects. Our results, while not calculated for $\mathrm{FeCoB}$, show that it is not required to invoke a mechanism via spin pumping but can arise due to an interplay between the anisotropy and the demagnetizing fields.

As well as looking at the effect of the film thickness on the damping parameter, we have also performed a systematic variation of the anisotropy constant. In FePt, the anisotropy can be modified, for example, by inducing lattice distortion 


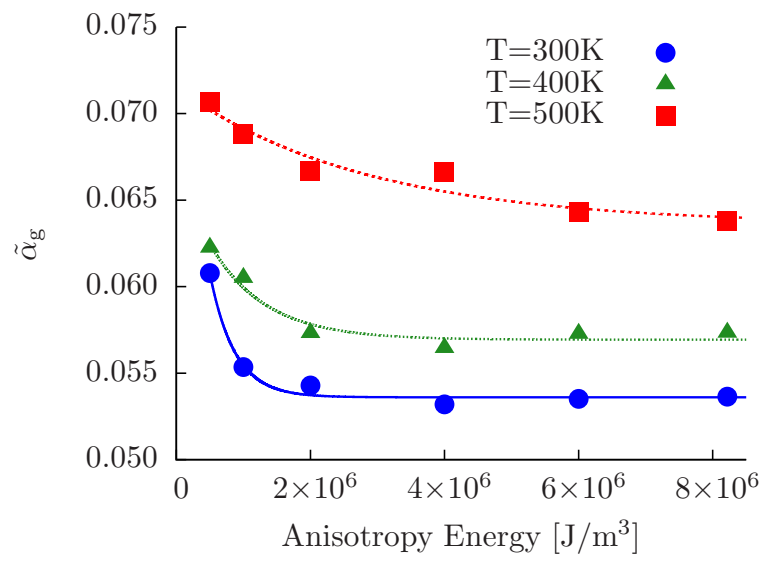

FIG. 6. (Color online) Dependence on the damping in FePt for a range of anisotropy constants for three values of temperature $(300 \mathrm{~K}$, blue circle points; $400 \mathrm{~K}$, green triangle points; and $500 \mathrm{~K}$, red square points). In the lower anisotropy range the damping increases, consistent with the results of Fig. 5. The lines are fits to exponential decays to give a guide to the eye.

or chemical disorder [40]. For the 2-nm-thick films, we have calculated the FMR spectra at three different temperatures $(300,400$, and $500 \mathrm{~K})$ for a range of anisotropy values below the bulk value (vertical dashed gray line in Fig. 5). From these calculations we have measured the effective damping parameters using the method described above. The overall trend shows a decrease in the measured damping, the result of which is shown in Fig. 6.

The overall trend in Fig. 6 shows a decrease in the damping when the anisotropy becomes dominant over the demagnetizing field, consistent with the results of Fig. 5.

Figure 7 shows the calculated switching times for four temperatures $(610,620,630$, and $640 \mathrm{~K})$ as a function of the thickness of the film. To calculate the switching times, we equilibrated the system at the temperature shown in the figure, we then applied a field with a step function to $2 \mathrm{~T}$ to reverse the magnetization in the $z$ direction. The switching times were then averaged over 25 runs per point. The errors in the switching times are quite small, so 25 runs seems to be a sufficient number to take a good average.

The thickness dependence of the switching times shown in Fig. 7 is consistent with the calculations of the damping presented in Fig. 5. As the thickness is increased, there is an observed decrease in the damping which leads to the reduced switching times seen in Fig. 7. It should be pointed out that the field that we apply is not sufficient to switch the magnetization below around $610 \mathrm{~K}$, consistent with Ref. [41]. The large reduction in the switching time seen for the $T=640 \mathrm{~K}$ case is due to the fact that with the inclusion of the stochastic term there is a slight reduction in the Curie temperature as shown in Ref. [16].

\section{CONCLUSION}

We have derived, using the LLB formalism, an analytic solution to the power frequency spectrum for nanometer-sized, single-domain ferromagnets during ferromagnetic resonance. Using the technologically relevant $\mathrm{FePt}$, this analytic solution

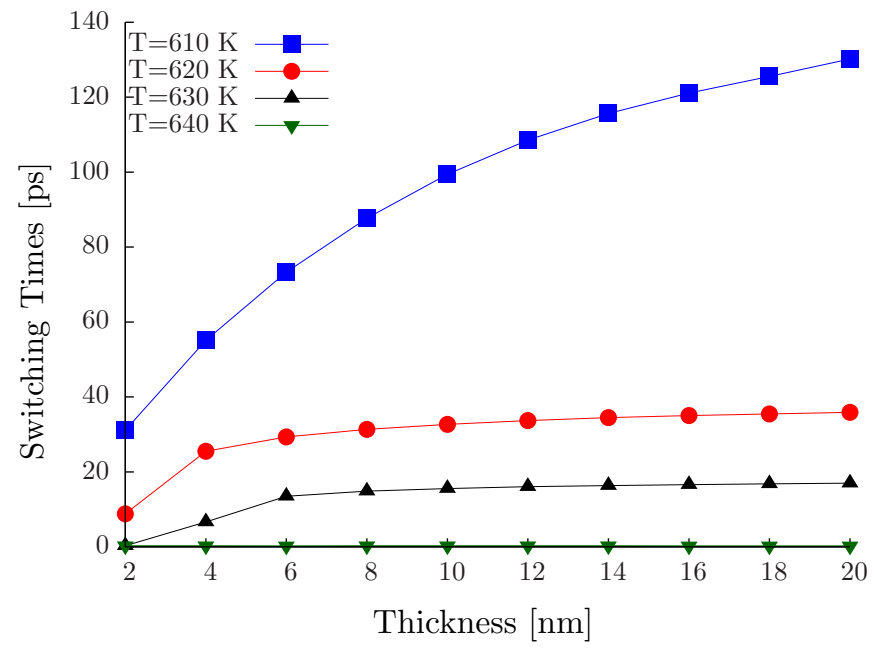

FIG. 7. (Color online) Switching times for thin films of FePt of differing thicknesses for a range of temperatures. Consistent with the result of Fig. 5, the reduction in the damping with increasing film thickness leads to an increase in the switching time. A Heaviside step function of $2 \mathrm{~T}$ was applied to the field to reverse the magnetization after equilibration and the runs were averaged over 25 realizations of the random number seed. With the inclusion of the stochastic term there is a reduced $T_{\mathrm{C}}$ so the $T=640 \mathrm{~K}$ line is already above the transition temperature.

agrees well with numerical simulations of both single-spin and exchange-coupled multispin calculations including the stochastic thermal term.

Analysis of the resulting FMR expressions for a single macrospin show that the analytically derived damping is consistent with those of extended thin films up to quite high temperatures. At temperatures approaching $T_{\mathrm{C}}$, the anisotropy decreases more rapidly than the magnetization. This leads to a region where demagnetizing field dominates over the anisotropy in the thin films. This means that our analytic expressions for thin films of magnetically soft materials would not hold, however, the analysis is still valid for single macrospins (or small structures) of soft materials.

We have extended the calculations to include the thermal stochastic term and demagnetizing effects to explore the effect this plays on the temperature-dependent ferromagnetic resonance curves. By calculating FMR spectra as a function of film thickness, we have shown that there is an increased damping for thinner films due to the interplay between the demagnetizing fields and the anisotropy. For the thinner films, there is more of a tendency for the magnetization to want to lie in plane due to the demagnetizing field. For highly anisotropic materials (shown here for FePt), this effect is more dominant at elevated temperatures. We have verified that this increase in damping can be explained by a change in the dominance of the demagnetizing energy by varying the anisotropy constant for the thin films. As the anisotropy constant is decreased the damping increases, consistent with the results of varying the film thickness.

Finally, we have shown that this reduction in the damping has an effect on the switching times. This conclusion could have important consequences for heat-assisted magnetic recording, which operates at elevated temperatures, and 
requires sufficiently thick grains to have sufficient material for good readback of the magnetic signal.

\section{ACKNOWLEDGMENTS}

This work was supported by the European Commission under Contract No. 281043, FemtoSpin. The financial support of the Advanced Storage Technology Consortium is gratefully acknowledged. The authors also thank R. W. Chantrell for helpful discussions.

\section{APPENDIX A: DETAILS OF ANALYTIC DERIVATION OF P $(\omega)$}

This section gives some more detail regarding the derivation of the key equations discussed in Sec. III. The linearized equations of motion for the LLB equation (1) are written as

$$
\begin{aligned}
\dot{m}_{x} \approx & -\gamma\left(m_{y} H_{\mathrm{eff}}^{z}-m H_{\mathrm{eff}}^{y}\right)+\frac{\gamma\left(\alpha_{\|}-\alpha_{\perp}\right)}{m}\left(m_{x} H_{\mathrm{eff}}^{z}\right) \\
& +\frac{\gamma \alpha_{\perp}}{m}\left(m H_{\mathrm{eff}}^{x}\right), \\
\dot{m}_{y} \approx & -\gamma\left(m H_{\mathrm{eff}}^{x}-m_{x} H_{\mathrm{eff}}^{z}\right)+\frac{\gamma\left(\alpha_{\|}-\alpha_{\perp}\right)}{m}\left(m_{y} H_{\mathrm{eff}}^{z}\right) \\
& +\frac{\gamma \alpha_{\perp}}{m}\left(m H_{\mathrm{eff}}^{y}\right), \\
\dot{m}_{z}= & 0,
\end{aligned}
$$

with the linearized effective fields then written as

$$
\begin{aligned}
\mathbf{H}_{\mathrm{eff}}^{x, y} & =B_{x, y}-\frac{m_{x, y}}{\tilde{\chi}_{\perp}}+\frac{1}{2 \tilde{\chi}_{\|}}\left(1-\frac{m^{2}}{m_{\mathrm{e}}^{2}}\right) m_{x, y}, \\
\mathbf{H}_{\mathrm{eff}}^{z} & =B_{z}+\frac{1}{2 \tilde{\chi}_{\|}}\left(1-\frac{m^{2}}{m_{\mathrm{e}}^{2}}\right) m .
\end{aligned}
$$

In equilibrium, the $z$ component of the effective field vanishes, $\mathbf{H}_{\mathrm{eff}}^{z}=0$. Using the linearized form of $m, m=m_{\mathrm{e}}(1+\Delta m)$ as well as $m^{2}=m_{\mathrm{e}}^{2}(1+2 \Delta m)$, we arrive at an expression for the $z$ component of the applied magnetic field

$$
B_{z}-\frac{m_{\mathrm{e}} \Delta m+m_{\mathrm{e}} \Delta m^{2}}{\tilde{\chi}_{\|}}=0 .
$$

Using the linearized form of this equation $B_{z}-\frac{m_{\mathrm{e}} \Delta m}{\tilde{\chi}_{\|}}=0$ as well as the approximation $\Delta m=\left(m-m_{\mathrm{e}}\right) / m_{\mathrm{e}}$, we have an approximation for $m$ during FMR that is both field and temperature dependent:

$$
m\left(T, B_{z}\right)=\tilde{\chi}_{\|}(T) B_{z}+m_{\mathrm{e}}(T) .
$$

As is discussed in the main text, the approximation (A3) leads to errors in the analytic treatment if the resonance curve is calculated in an applied field. This is due to the fact that the susceptibility diverges as we approach the Curie temperature. This does not occur in the numerical simulations and is only a problem in the analytic calculations due to the above approximation (A3).

In order to calculate the resonance frequency $\left(\omega_{0}\right)$ as well as the transverse relaxation time $(\tau)$ for the power spectrum $P(\omega)$, one has to solve the linearized LLB equation [see Eq. (A1)]. Using the notation $\tilde{m}=m_{x}+i m_{y}$ and $\tilde{H}_{\mathrm{eff}}=H_{\mathrm{eff}}^{x}+i H_{\mathrm{eff}}^{y}$ leads to the differential equation

$$
\frac{\dot{\tilde{m}}}{\gamma}=\tilde{m}\left(i+\frac{\alpha_{\|}-\alpha_{\perp}}{m}\right) H_{\mathrm{eff}}^{z}+m\left(\frac{\alpha_{\perp}}{m}-i\right) \tilde{H}_{\mathrm{eff}} .
$$

As can be easily seen from Eq. (A2), $\tilde{H}_{\text {eff }}$ is also $\tilde{m}$ dependent. Writing the effective field as $\tilde{H}_{\text {eff }}=\tilde{B}+A \tilde{m}$, with $A=-\frac{1}{\tilde{\chi}_{\perp}}+\frac{1}{2 \tilde{\chi}_{\|}}\left(1-\frac{m^{2}}{m_{\mathrm{e}}^{2}}\right)$ and $\tilde{B}=B_{x}+i B_{y}$ we arrive at an inhomogeneous differential equation

$$
\begin{aligned}
\frac{\dot{\tilde{m}}}{\gamma}= & \tilde{m}\left[\left(i+\frac{\alpha_{\|}-\alpha_{\perp}}{m}\right) H_{\mathrm{eff}}^{z}\right]+\tilde{m}\left[m\left(\frac{\alpha_{\perp}}{m}-i\right) A\right] \\
& +m\left(\frac{\alpha_{\perp}}{m}-i\right) \tilde{B} .
\end{aligned}
$$

In the first step, we solve the homogeneous part of the differential equation (A4), using the ansatz $\tilde{m}_{\text {hom }}(t)=\exp (\omega t)$ whose solution leads to the expressions for $\omega_{0}$ and $\tau$ :

$$
\begin{aligned}
\omega_{0} & =\gamma\left(B_{z}+\frac{m}{\tilde{\chi}_{\perp}}\right), \\
\tau & =\frac{m}{\lambda\left[\left(1-\frac{T}{3 T_{\mathrm{c}}}\right) \omega_{0}-\frac{2}{3} \gamma \frac{T}{T_{\mathrm{c}}} H_{\mathrm{eff}}^{z}\right]} .
\end{aligned}
$$

In the next step, we solve the inhomogeneous differential equation (A4) under the assumption that the applied magnetic field $\mathbf{B}$ has the form $\mathbf{B}=\left[B_{0} \exp (i \omega t), 0, B_{z}\right]$, where $B_{0} \ll B_{z}$. These lead to the following simplification of the right-hand side of Eq. (A4):

$$
m\left(\frac{\alpha_{\perp}}{m}-i\right) \tilde{B}=m\left(\frac{\alpha_{\perp}}{m}-i\right) B_{0} \exp (i \omega t) .
$$

Using the ansatz $\tilde{m}(t)=u(t) \tilde{m}_{\text {hom }}(t)$ where $u(t)$ is given by

$$
u(t)=\int_{t_{0}}^{t} \frac{m\left(\frac{\alpha_{\perp}}{m}-i\right) B_{0} \exp (i \omega t)}{\exp \left(-\frac{t}{\tau}\right) \exp \left(i \omega_{0} t\right)} d t
$$

and assuming $t_{0}=0$ and $t \rightarrow \infty$, Eq. (A4) has the solution

$$
\tilde{m}(t)=\frac{\left(-i+\frac{\alpha_{\perp}}{m}\right) \gamma m B_{0}\left[\frac{1}{\tau}-i\left(\omega-\omega_{0}\right)\right]}{\frac{1}{\tau^{2}}+\left(\omega-\omega_{0}\right)^{2}} \exp (i \omega t) .
$$

From this general solution, $m_{x}$ can easily be derived

$$
\begin{aligned}
m_{x}= & \frac{\gamma m B_{0}}{\frac{1}{\tau^{2}}+\left(\omega-\omega_{0}\right)^{2}}\left[\left(\frac{\alpha_{\perp}}{\tau m}-\left(\omega-\omega_{0}\right)\right) \cos (\omega t)\right. \\
& \left.+\left(\frac{1}{\tau}+\frac{\alpha_{\perp}}{m}\left(\omega-\omega_{0}\right)\right) \sin (\omega t)\right],
\end{aligned}
$$

and substituted into the definition for the power spectrum $P(\omega)$ [see Eq. (6)]. This leads to the analytic solution for the power spectrum $P(\omega)$ :

$$
P(\omega)=\frac{M_{\mathrm{s}} V \omega^{2}}{4} \frac{\gamma \alpha_{\perp} B_{0}^{2}}{\frac{1}{\tau^{2}}+\left(\omega-\omega_{0}\right)^{2}} .
$$

As we can see from Eq. (A9), the analytic solution for the absorbed power depends on the magnetization, which in turn depends on the longitudinal susceptibility. As mentioned above, we approximate the magnetization in the presence of an applied field [Eq. (A3)] in terms of the zero-field susceptibility. Therefore, Eq. (A3) is only strictly correct in the zero-field limit. Away from the critical temperature, the 


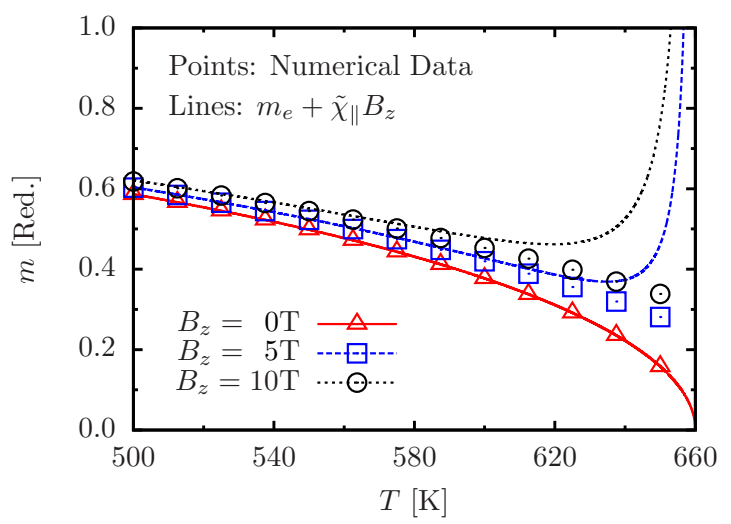

FIG. 8. (Color online) Equilibrium magnetization vs temperature in different applied fields. The (red) solid line represents the zero-field equilibrium magnetization $m_{\mathrm{e}}$ gained from atomistic $\mathrm{FePt}$ simulations, a fit to which defines the input function $m_{e}(T)$ [17]. The dashed (blue) and dotted (black) lines represent expression (A3) for different applied fields. The symbols represent the equilibrium magnetization in the presence of an applied field $B_{z}=0,5,10 \mathrm{~T}$, from the numerical simulations of a single macrospin without demagnetizing, stochastic, or exchange fields.

zero-field susceptibility is small, therefore, in this limit the approximation holds. As we approach the critical temperature, the susceptibility diverges as we approach the phase transition. This means that our analytic expression shows a deviation from the numerically calculated result.

A plot of the magnetization as a function of temperature using Eq. (A3) and data from numerical simulations can be seen in Fig. 8. For small values of the applied field, this error reduces as the susceptibility is defined for small changes in the applied field.

Figure 8 shows the equilibrium magnetization (red solid curve), initially calculated from atomistic spin dynamics simulations [17], which is used as an input to the numeric simulation. As well as the equilibrium magnetization, the figure also shows the magnetization as a function of temperature in 5- and 10-T applied fields, which is of course not zero at the (zero-field) Curie temperature. The dashed and dotted line is the analytic solution to the magnetization (also in 5and 10-T fields), diverging across the Curie temperature. As we can see, the magnetization in an applied field from the analytic expression shows a diverging behavior as we approach the Curie temperature because of the diverging susceptibility, whereas the numerical simulations (points) show no such divergence.

\section{APPENDIX B: ANALYTIC FERROMAGNETIC RESONANCE CURVES FOR FIXED FREQUENCY}

As was pointed out in the main text, the experimentally more easily accessible measurement involves keeping the driving frequency fixed (usually around $10-60 \mathrm{GHz}$ ) and varying the applied magnetic field until the resonance condition is met. The representation that we have used in our calculations and the analytic solutions that we have shown in the main paper keeps the applied field constant at $1.0 \mathrm{~T}$ in the $z$ direction and varies the frequency. The reason for using this representation

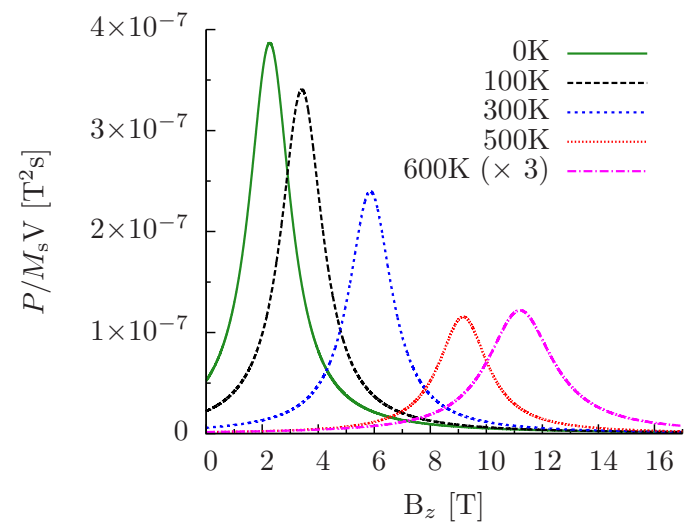

FIG. 9. (Color online) Power absorbed during ferromagnetic resonance for a fixed frequency of the driving field of $504.2 \mathrm{GHz}$ as a function of the applied field $B_{z}$. The curves are given by the analytic solution [Eq. (9)] and are shown for a range of temperatures. There is an increasing field required to drive the system to resonance. Note that the $T=600 \mathrm{~K}$ curve has been scaled for clarity as shown in the legend.

is that for the case of a large number of macrospins coupled by exchange and demagnetizing fields, the variation of the frequency is computationally more efficient. Therefore, to be consistent between our results and the subsequent analysis we also presented the analytic solutions and single macrospin calculations (which would be easily calculated with a fixed frequency) in the same representation $[P(\omega)]$.

It should be pointed out that experimentally measuring FMR for FePt is quite difficult in general (as was pointed out in Ref. [18]) it has not been possible (to our knowledge) to measure FMR in ordered $\mathrm{L} 1_{0} \mathrm{FePt}$ due to its high anisotropy, particularly at low temperatures. As was also shown in Ref. [19], the resonance frequency is in the hundreds of $\mathrm{GHz}$ regime for FePt with a high degree of ordering. In Fig. 9, we have shown Eq. (9) for a fixed frequency and varied the applied field, essentially giving us $P\left(B_{z}\right)$. Due to the extremely large magnetocrystalline anisotropy in $\mathrm{FePt}$, the use of driving frequencies of $10-60 \mathrm{GHz}$ would show negative resonance fields (the field required to drive the system to resonance). For our single macrospin analytic approximation at $0 \mathrm{~K}$, the frequency required to give zero resonance field would be just below $450 \mathrm{GHz}$.

Figure 9 shows the results of the analytic solution [Eq. (9)] for a fixed value of the driving frequency $(\omega=504.2 \mathrm{GHz})$ as a function of the applied field. The data are shown for a range of temperatures and show that as the temperature is increased, the value of the field required to drive the system to resonance increases consistent with a reduction in the anisotropy as seen in Refs. [14,34]. For room-temperature values of the temperature, the field required to drive the system to resonance is quite large (around $6 \mathrm{~T}$ ). Note that the value of the driving frequency of $504.2 \mathrm{GHz}$ was chosen so that a reasonable positive resonance field was required to drive the system to resonance at lower temperatures. The data of Fig. 9 would give a qualitatively similar result for a lower frequency but with a shifted set of resonance fields shifted in the negative field range. 


\section{APPENDIX C: MAGNETOSTATIC FIELDS}

For efficient calculation of the magnetostatic fields we write the convolution (13) as

$$
H_{d, i}^{\eta}=\sum_{\theta, j} W_{i j}^{\eta \theta} m_{j}^{\theta},
$$

where the greek symbols $\eta, \theta$ again denote Cartesian components $x, y, z$ and latin symbols $i, j$ denote the lattice sites. $W_{i j}^{\eta \theta}$ are interaction matrices which only depend on the structure of the material (cubic in this work). Since we are considering a translationally invariant lattice, one can apply the discrete convolution theorem and calculate the fields in Fourier space:

$$
H_{d, k}^{\eta}=\sum_{\theta} W_{k}^{\eta \theta} m_{k}^{\theta}
$$

It should be pointed out here that we have absorbed the prefactor $M_{s}$ into the interaction matrix $W_{i j}^{\eta \theta}$. Furthermore, to write the fields in terms of units of Tesla to be consistent with the form of the fields above, we have multiplied Eq. (13) by $\mu_{0}$. The Fourier transform of the interaction matrix only has to be performed once and thus stored in memory.

There are a number of conditions that must be met in order to utilize the convolution theorem. In terms of signal processing theory, the interaction matrix is seen as the response function and the magnetization data is the signal. We should note that there are two conditions that must be satisfied to utilize the convolution theorem. The first is that the signal (spin system) must be periodic in space. The second is that the range of the response function should be the same as the signal [42]. The magnetic system is usually not periodic and the demagnetizing effects are long ranging and cannot be cut off at a reasonable distance due to the slow decay [42]. To solve this, we simulate a finite system, therefore, to meet the above requirements it is required that we zero pad the magnetization configurations by doubling the size of each dimension and adding zeros in the areas where there are no macrospins.

At each update of the demagnetizing field (every $10 \mathrm{fs}$ ), the Fourier transform of the magnetization arrays is performed and the resulting Fourier components convoluted with that of the interaction matrix. The resulting product is back transformed via an inverse Fourier transform to give the demagnetizing fields in real space.
[1] T. W. Clinton, N. Benatmane, J. Hohlfeld, and E. Girt, J. Appl. Phys. 103, 07F546 (2008).

[2] A. J. Schellekens, L. Deen, D. Wang, J. T. Kohlhepp, H. J. M. Swagten, and B. Koopmans, Appl. Phys. Lett. 102, 082405 (2013).

[3] P. Krone, M. Albrecht, and T. Schrefl, J. Magn. Magn. Mater. 323, 432 (2011).

[4] A. Sukhov, K. Usadel, and U. Nowak, J. Magn. Magn. Mater. 320, 31 (2008).

[5] S. Sun, Science 287, 1989 (2000).

[6] R. P. Cowburn, D. K. Koltsov, A. O. Adeyeye, M. E. Welland, and D. M. Tricker, Phys. Rev. Lett. 83, 1042 (1999).

[7] J. L. Dormann, D. Fiorani, and E. Tronc, Advances in Chemical Physics (Wiley, Hoboken, NJ, 2007), pp. 283-494.

[8] M. Farle, Rep. Prog. Phys. 61, 755 (1998).

[9] M. O. A. Ellis, T. A. Ostler, and R. W. Chantrell, Phys. Rev. B 86, 174418 (2012).

[10] D. Weller and A. Moser, IEEE Trans. Magn. 35, 4423 (1999).

[11] M. Kryder, E. Gage, T. McDaniel, W. Challener, R. Rottmayer, and M. Erden, Proc. IEEE 96, 1810 (2008).

[12] N. Smith and P. Arnett, Appl. Phys. Lett. 78, 1448 (2001).

[13] S. Maat, N. Smith, M. J. Carey, and J. R. Childress, Appl. Phys. Lett. 93, 103506 (2008).

[14] K. D. Usadel, Phys. Rev. B 73, 212405 (2006).

[15] N. Minnaja, Phys. Rev. B 1, 1151 (1970).

[16] R. F. L. Evans, D. Hinzke, U. Atxitia, U. Nowak, R. W. Chantrell, and O. Chubykalo-Fesenko, Phys. Rev. B 85, 014433 (2012).

[17] N. Kazantseva, D. Hinzke, U. Nowak, R. W. Chantrell, U. Atxitia, and O. Chubykalo-Fesenko, Phys. Rev. B 77, 184428 (2008).

[18] N. Álvarez, G. Alejandro, J. Gómez, E. Goovaerts, and A. Butera, J. Phys. D: Appl. Phys. 46, 505001 (2013).
[19] J. Becker, O. Mosendz, D. Weller, A. Kirilyuk, J. C. Maan, P. C. M. Christianen, T. Rasing, and A. Kimel, Appl. Phys. Lett. 104, 152412 (2014).

[20] A. Butera, Eur. Phys. J. B 52, 297 (2006).

[21] D. A. Garanin, Phys. Rev. B 55, 3050 (1997).

[22] J. Kötzler, D. A. Garanin, M. Hartl, and L. Jahn, Phys. Rev. Lett. 71, 177 (1993).

[23] N. Kazantseva, R. Wieser, and U. Nowak, Phys. Rev. Lett. 94, 037206 (2005).

[24] O. Chubykalo-Fesenko, U. Nowak, R. W. Chantrell, and D. Garanin, Phys. Rev. B 74, 094436 (2006).

[25] R. F. L. Evans, W. J. Fan, P. Chureemart, T. A. Ostler, M. O. A. Ellis, and R. W. Chantrell, J. Phys.: Condens. Matter 26, 103202 (2014).

[26] O. N. Mryasov, U. Nowak, K. Y. Guslienko, and R. W. Chantrell, Europhys. Lett. 69, 805 (2005).

[27] J. Mendil, P. Nieves, O. Chubykalo-Fesenko, J. Walowski, T. Santos, S. Pisana, and M. Münzenberg, Sci. Rep. 4, 3980 (2014).

[28] U. Nowak, O. N. Mryasov, R. Wieser, K. Guslienko, and R. W. Chantrell, Phys. Rev. B 72, 172410 (2005).

[29] D. Hinzke, U. Nowak, R. W. Chantrell, and O. N. Mryasov, Appl. Phys. Lett. 90, 082507 (2007).

[30] D. Hinzke, N. Kazantseva, U. Nowak, O. N. Mryasov, P. Asselin, and R. W. Chantrell, Phys. Rev. B 77, 094407 (2008).

[31] J. Becker, O. Mosendz, D. Weller, A. Kirilyuk, J. C. Maan, P. C. M. Christianen, Th. Rasing, and A. Kimel, Appl. Phys. Lett. 104, 152412 (2014).

[32] T. A. Ostler, R. F. L. Evans, R. W. Chantrell, U. Atxitia, O. Chubykalo-Fesenko, I. Radu, R. Abrudan, F. Radu, A. Tsukamoto, A. Itoh, A. Kirilyuk, T. Rasing, and A. V. Kimel, Phys. Rev. B 84, 024407 (2011). 
[33] N. Kazantseva, Ph.D. thesis, The University of York, 2008.

[34] B. Schulz and K. Baberschke, Phys. Rev. B 50, 13467 (1994).

[35] C. Antoniak, J. Lindner, and M. Farle, Europhys. Lett. 70, 250 (2005).

[36] L. Lopez-Diaz, D. Aurelio, L. Torres, E. Martinez, M. a. Hernandez-Lopez, J. Gomez, O. Alejos, M. Carpentieri, G. Finocchio, and G. Consolo, J. Phys. D: Appl. Phys. 45, 323001 (2012).

[37] A. J. Newell, W. Williams, and D. J. Dunlop, J. Geophys. Res. 98, 9551 (1993).
[38] M. J. Donahue and D. G. Porter, OOMMF User's Guide, Version 1.0, NISTIR 6376, National Institute of Standard and Technology, Gaithersburg, Maryland, United States, 1999, http://math.nist.gov/oommf.

[39] X. Liu, W. Zhang, M. J. Carter, and G. Xiao, J. Appl. Phys. 110, 033910 (2011).

[40] C. J. Aas, L. Szunyogh, J. S. Chen, and R. W. Chantrell, Appl. Phys. Lett. 99, 132501 (2011).

[41] N. Kazantseva, D. Hinzke, R. W. Chantrell, and U. Nowak, Europhys. Lett. 86, 27006 (2009).

[42] D. V. Berkov and N. L. Gorn, Phys. Rev. B 57, 14332 (1998). 